\title{
RESPONSES OF SINGLE AUDITORY UNITS TO RANDOM STIMULATION AND LATERAL INHIBITION
}

\author{
N.G. BIBIKOV \\ N.N. Andreev Acoustical Institute, Moscow, USSR
}

\begin{abstract}
Cross-covariance functions (CGFs) between the spike sequence and the low-frequency notse used for the modulation of characteristic frequency (CF) and off-CF tones were obtained for cochlear rucleus neuxons of unanesthetized lake fross . The analysis of GaFs demoristrates the existing of the inhibitory inputs which seem to enlarge the dynamic range of amplitude modulation coding in secondary auditory units comparing with primary ones.
\end{abstract}

The single secondary-arder neurors of the auditory system process the inputs they receive from the auditory nerve ribers. one of the main differences between the spiral sanglion cells and the cochlear nucleus units is the fidelity with which changes in sound amplitude are encoded. The auditory nerve fibers reproduce the stimulus amplitude variationswithout any corssiderable amplification. Moreover, this reproduction takes place only in the restricted dynamic operation range: $30-40$ dB above the threshold /1/. In contrast the majority of the cochlear mucleus units demonstrate the amplificiation of the stimulus modulation depth and this amplification is observed over a great dynamic range $/ 1,2 /$.

It is not difficult to explain the widening of the operation rarge by the conversency of many excitatory inputs on one cochlear nucleus cell. The fibers which provided these inputs car have different thresholds and different characteristic frequerscies. The summed input excitation would be increased by sound amplitiude increment resardless of how intense an inttial amplitude was.

This mechanism can not, however, explain many important. features of the amplitude modulation encodins in cochlear rucleus cells. First of all it doesn't explain the amplification of the stimulus amplitude changes in cochlear nucleus units. On the contrary the relative weight, of the input changes evoked by a fixed sound increment has to be decreased as a result of the convergency of many fibers with various thresholds and various dynamic ranses. Also, the rate-intersity runction for stationary tone whould have the same operation range as that for encoding of small amplitude changes. It is not the case for the majority of the cochlear nucleus. The operation ranse for modulation encoding as a rule exceeds that of the average rate-intensityfunction considerably $/ 2 /$. Finally, the convergency of the excitator.y inputs with different characteristic frequencies must lead to the considerable widening of the tuning curve and the worsening of the frequency selectivity. Irideed in cochlese nucleus nemras some widenine of the tip of the turing curve is observed, but theiv. 
edges are usually as sharp as those in auditory nerve ribers. Moreover in many cochlear mucleus cells a low-rrequency tall of the tuning curve is absent $/ 3 /$.

Mast of these difficulties, however, could be override if the hypothesis of how cochlear nucleus neuroris enhance the amplitude modulation information will include the delayed lateral inhibition influences. The amplification of the modulation can be explained by relative decreasing of the average impulsation rate and by the enhancement of the reaction to amplitude chanses. The enlargement. of the operation sange for amplitude-modulation encodins may be treated as a dixect consequence of the time difference between the exatatory and inhibitory inputs. For intense stimuli the steady-state rate-intensity runction is flat. The stimulus increment, however, causes the initial excitatory input that evokes the spike seneration. The succeeded inhibition can not prevent the reaction to the increment. Finally, the absence of the considerable widening of the tuning curves in secondary- order units is easily explained if we assume that the inhibitory inputs come from the larger region of the cochlea than the exattatory ones. It is well known that many cochlear nucleus neurons exhibit strong inhibitory components in their responses to of $\mathrm{f}$-characteristic frequency (CF) tone bursts $/ 4 /$.

In our study we have investifated the dynamic properties of the responses of cochleax rucleus neurons in unanesthetized curarized lake frogs (Rana ridibunda). The stimuli were lons-duration (100 sec) tones of different intensities and rrequencies. They were amplitude-modulated by random low-frequency $(0-200$ Hz) noise with the modulation depth ( root mean square) - 16\%. The dynamic properties of the cells were described by cross-covariance fumctions (CGF) between the recorded discharge sequence and the modulation vaveform.

The detalls of the methodolagy are described in our previous paper/s/. The sound delivering system was closed and stimull were delivered on the ipsilateral ear. The registration was extracellular and glass micropipettes filled with 3 M Nacl were used. We used the standard equipment for stimulus generation, control and calibration. The spike activity was ampliried, trisfered and fed to a ATAC 501-20 CNihon Kohdens analyzer. The CGFs were obtained by synchronized summation af the stimulus envelope waverorms that preceded the spikes sereration. In contrast to Moller's experiments $/ 1,6,7 /$ we used for modulation not pseudorandom noise but continuous random noise with saussian amplitude distribution..

The set of the CGFs obtained in typical cochlear nucleus cells when the carrier was the tone at unit's characteristic frequency is shown in Fig 1 . With the low-intensity carriers (lower curves) the CCFs have only a single positive peak. Such shape of the CCF indicates that a brief increment in stimulus intensity fives rise to an elevation in the neuron discharge rate. When the mean stimulus intensity is increased a negative deflection is usually appeared after this positive CCF peak. In some neurons at high intensities this deflection determines the whole shape of the chF.

We obtained the relative values of the CGF peaks by the dividing of peaks amplitude upon the mean impulsation rate. In fig 2 these relative peak values are shown as a function of carrier intensities for two cochlear nualeus units. When the carrier intersity increases the relative value of the positive caf peak saturates 10 dB above threshold. The relative value of the negative peak, however, monotonically increases through the whole investifated interusity range. Thus the role or the delayed inhibitory input. increases with the intensity of the ch tone. 

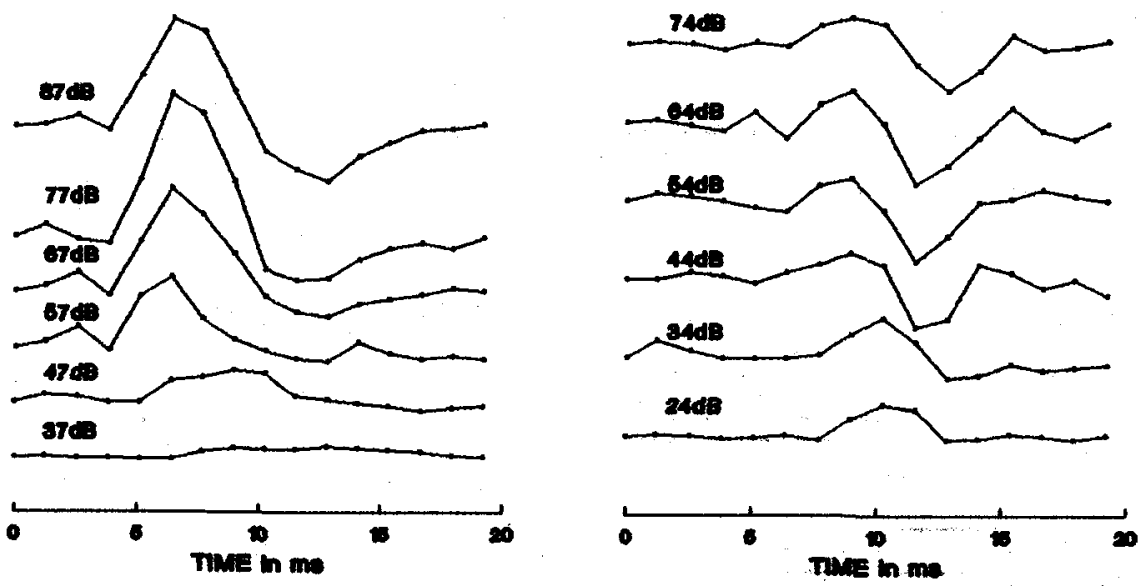

Fig.1. Cross-covariance functions for two units in response to $G F$ tone modulated by $200 \mathrm{~Hz}$ low-pass noise. The carrier intensity is shown near the curves in dB re unit threshold. Neuron 80-10-5 (left row): GF $0.8 \mathrm{kHz}$; threshold 32 dB SPL. Neuron 81-6-3 cright row): GF $0.65 \mathrm{kHz}$; threshold $19 \mathrm{~dB}$ SPL.
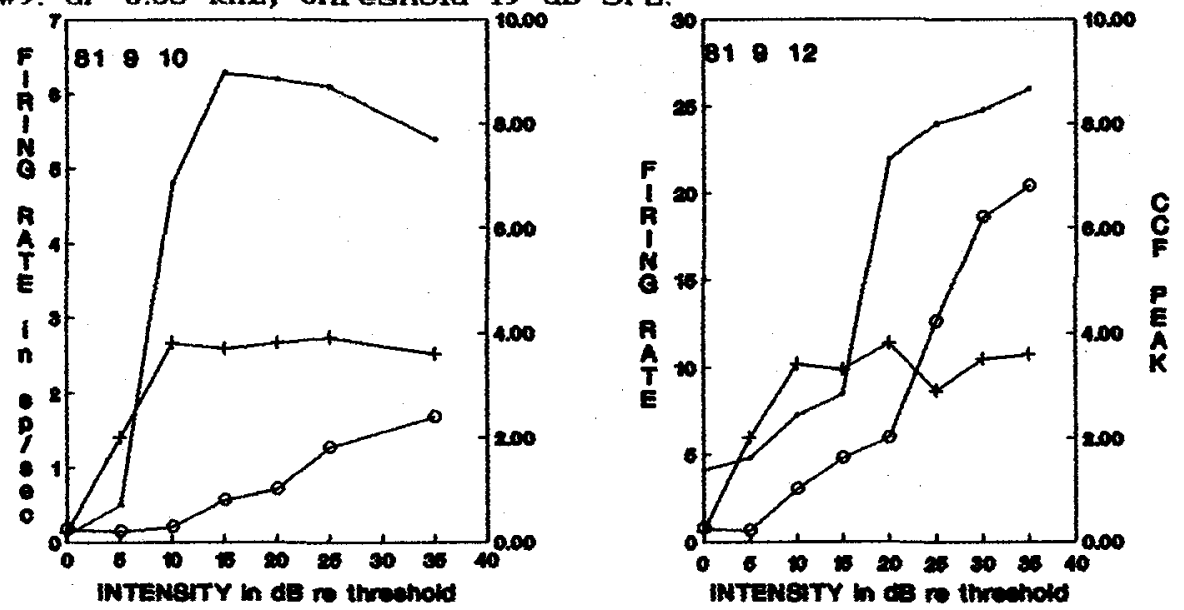

Fig 2. Mean impulsation rates (rilled circles), positive CGF peak amplitudes (circlés) and negative CCF peak amplitudes (crosses) are plotted for two units as a function of the carrier intensity ir dB re units threshold. The values of the GGF peaks are shown in relative values. Neuron $81-9-10$ Cleft row): GF $0,8 \mathrm{kHz}$; threshold 43 dB SPL. Neumon 81-9-12 (right row): CF $1.2 \mathrm{kHz}$; threshold $54 \mathrm{~dB}$.

The role of the inhibitory inputs becomes mare evident when off-CF tones are used as a carrier. The set of the CCFs obtained in typical cochlear nucleus cell at various carrier rrequencies is shown in Fif 3 . With the best-rrequency carriers $(0,5 \mathrm{kHz})$ the CCF reveals a positive peak that succeeded by a negative one. The shape of the CGF is radically changed when the carrier frequency moves far enough from the CF. For $0.3 \mathrm{kHz}$ tone it is the negative CGF peak that preceded the positive one. At the edses of the frequency response range $(0.2 \mathrm{kHz}$ and $0.9 \mathrm{kHz})$ the negative peak 

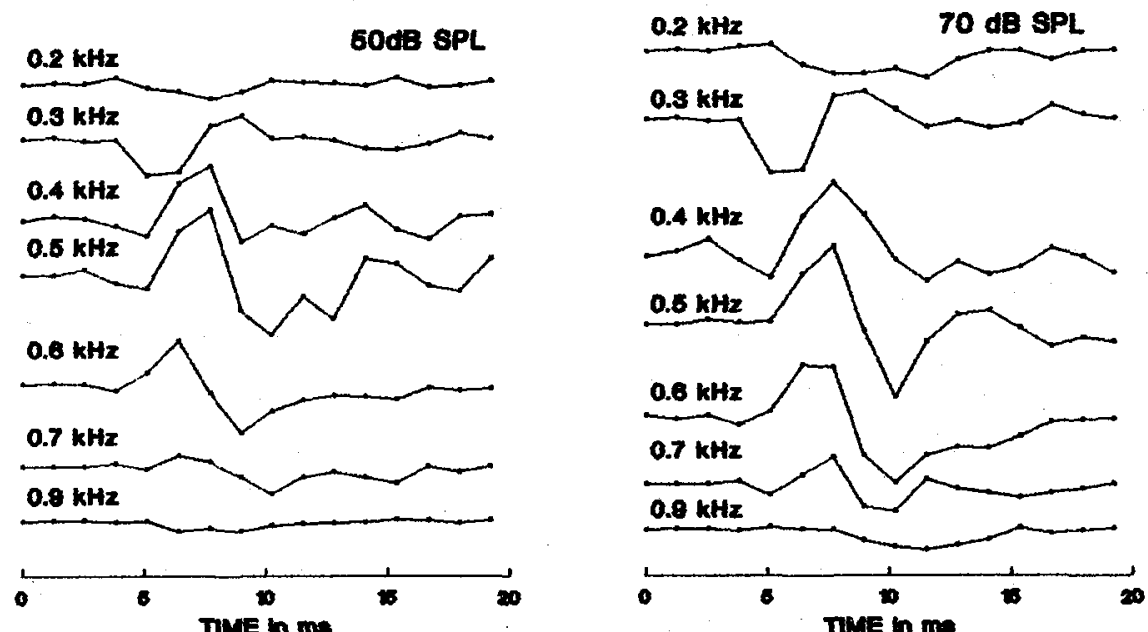

Fis.3. Cross-covariance functions at two intensity levels 650 dB SPL - left row; 70 dB SPL - right row obtalned for tones modulated by $200 \mathrm{~Hz}$ low-pass noise. The carrier frequencies in kHz are shown rear the curves. Neuron 80-13-18: CF - 0,5 kHz, threshold-36 dB SPL.

remains the only notable component of the CCF. Such shape of the GCF indicates that for these frequencies this neuron generates the spike discharges in response not to the increment but in fact to the decrements of the sound envelope. It is of interest that the mean impulsation rate for amplitude modulated off-cF tones which evoke such "off-response" can be fairly hish.

The analysis of CCFs obtained in cochlear nucleus neurors of the frog allows to susfest that the complex interaction of the excitatory and inhibitory inputs takes place during the action of the amplitude-modulated CF and off-CF tones. For high intensity CF tones delayed negative peak of GaF could demonstrate the delayed inhibitory input. For off-GF tones this inhibitory input becomes the leading one. Now it may be treated as a typical representation of lateral inhibition mechanisms. These findings sugsest that the same under-lyins cellular mechanism may be responsible far the lateral inhibition and the delayed GF-inhibition. The latter. effect can be used for the explanation of the enormous operation range for amplitude-modulation encoding in many cochiear nucleus neuroons.

Some madels of cochlear nucleus neurons assume independent inhibitory system which participate in response-stxength limitation. From the other hand the lateral inhibition is usually treated as a response property that improves the rrecuency selectlvity by the limitation of the excitatory response area. Oux evidence allow to sugsest the important role of the lateral inhibition both in the response-strength limitation and in the enlargement of the dynamic operation range during the action of complex modulated sounds.

1.MOLLER AR, Acta Physiol. Scand.98 (1975) 157-167.

2.FRISINA,R, SMTH,R, GHAMBERLAIN S, Hear. Res. 44 (1990) $123-142$. 3.RHODE, $W$, Smith $P$, J. Neurophysiol. 56 (1985) 287-307.

4.MARTIN,M, DICKSON,JW, Hear.Res., 9,(1983) 35-42.

5.BIBIKOV NG, Sensory. Systems, 1 (1987) 353-361.

6MOLLER AR ,Physiol 259. (1975) 63-82.

7.MOLLER AR Exp. Brain Res. 25 (1976) 307-321. 